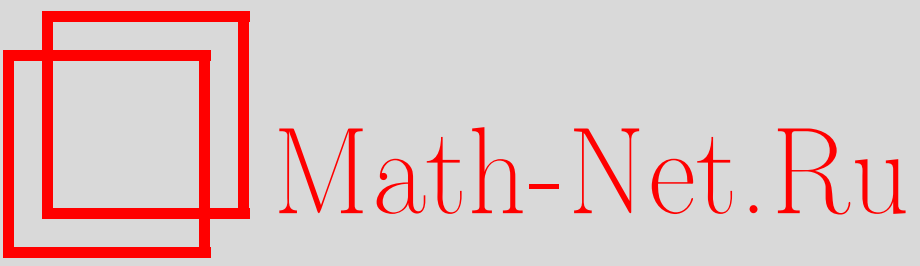

С. А. Никитина, В. И. Ухоботов, Дискретная линейная задача управления с терминальным множеством в форме кольца при наличии помехи, Итоги науки и техн. Сер. Соврем. мат. и ее прил. Темат. обз., 2020, том 186, 102-107

DOI: https://doi.org/10.36535/0233-6723-2020-186-102-107

Использование Общероссийского математического портала Math-Net.Ru подразумевает, что вы прочитали и согласны с пользовательским соглашением

http: //www. mathnet.ru/rus/agreement

Параметры загрузки:

IP : 54.166 .219 .16

26 апреля 2023 г., 13:58:30 


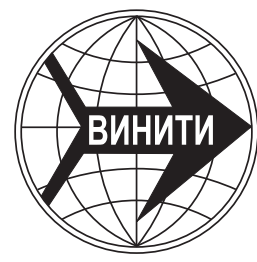

ИТОГИ НАУКИ И ТЕХНИКИ.

Современная математика и ее приложения.

Тематические обзоры.

Том 186 (2020). C. 102-107

DOI: 10.36535/0233-6723-2020-186-102-107

УДК 517.977

\title{
ДИСКРЕТНАЯ ЛИНЕЙНАЯ ЗАДАЧА УПРАВЛЕНИЯ С ТЕРМИНАЛЬНЫМ МНОЖЕСТВОМ В ФОРМЕ КОЛЬЦА ПРИ НАЛИЧИИ ПОМЕХИ
}

\author{
(C) 2020 г. \\ с. А. НИКИТИНА, В. и. УХОБОТОВ
}

\begin{abstract}
АннотАция. В статье рассматривается дискретная динамическая управляемая система с помехой. Требуется, чтобы в момент окончания процесса управления фазовая точка содержалась в заданном множестве, имеющем форму кольца. Записан оператор программного поглощения, с помощью которого сформулированы условия на множество начальных положений, при которых гарантируется выполнение требуемого включения в заданный момент времени.
\end{abstract}

Ключевые слова: дискретная система, задача управления, терминальное множество в форме кольца.

\section{DISCRETE LINEAR CONTROL PROBLEM WITH THE RING-SHAPED TERMINAL SET IN THE PRESENCE OF NOISE}

\author{
(c) 2020 S. A. NIKITINA, V. I. UKHOBOTOV
}

\begin{abstract}
In this paper, we consider a discrete dynamic control system with noise. It is required that at the terminal moment of process the phase point is contained in a given ring-shaped set. We find the operator of program absorption, which allows one to formulate conditions for the set of initial positions that guarantee the fulfillment of the required inclusion at a given moment of time.
\end{abstract}

Keywords and phrases: discrete system, control problem, ring-shaped terminal set.

AMS Subject Classification: 93C41, 93C55

1. Введение. В работе [5] рассмотрена однотипная дифференциальная игра с геометрическими ограничениями на управления игроков и с фиксированным моментом окончания, в которой терминальное множество имеет форму кольца. Были записаны правила выполнения операций суммы и геометрической разности кольца и шара в $n$-мерном пространстве.

В статье [6] для линейной дифференциальной игры импульсной встречи получен максимальный стабильный мост, ведущий в конечный момент времени на терминальное множество, заданное в форме кольца. Стабильный мост определяется функциями внешнего и внутреннего радиусов, которые вычислены в явном виде. В [7] для данной игры были построены оптимальные управления игроков. В [1] предложен новый подход к построению управления первого игрока, который позволяет сократить вычислительные процедуры.

В работе [8] рассмотрена дискретная линейная задача управления с помехой и одномерной целью. Условие одномерности означает, что модуль значения заданной линейной функции от 
фазовых переменных в фиксированный момент окончания процесса управления не должен превосходить заданного числа. Для этой задачи получены необходимые и достаточные условия, выполнение которых гарантирует существование допустимого управления, которое обеспечивает достижение цели при любой допустимой реализации помехи.

В статье [2] рассмотрена задача управления дискретной линейной системой для случая, когда терминальное множество и вектограмма управления являются многогранниками специального вида, которые задаются с помощью системы линейных неравенств. Получены условия на множество начальных положений, при которых гарантируется достижение цели в момент окончания процесса управления.

В данной статье продолжается исследование, начатое в указанных работах. Рассмотрена дискретная модель управляемого процесса с фиксированным моментом окончания, в которой терминальное множество имеет форму кольца.

Дискретные процессы управления возникают, как правило, при решении прикладных задач [4, 9]. Это связано с тем, что зачастую информация о состоянии процесса поступает в дискретные моменты времени, а управление осуществляется по шагам.

2. Пример. Вначале рассмотрим в качестве примера задачу управления запасами [4]. Предположим, что некоторое производственное объединение может выпускать товары двух видов.

Пусть $x(k)=\left(x_{1}(k) ; x_{2}(k)\right)$ - вектор количества выпускаемых товаров первого и второго вида, имеющихся в наличии на складе к концу $k$-го периода, $u(k)=\left(u_{1}(k) ; u_{2}(k)\right)$ - вектор интенсивностей (скорость производства), $s(k)=\left(s_{1}(k) ; s_{2}(k)\right)$ - вектор количества товаров, отгруженных со склада в $k$-й период (определяется спросом на товары).

Уравнение, описывающее дискретный процесс, имеет вид

$$
x(k+1)=x(k)+u(k)-s(k), \quad k=0,1, \ldots, N-1 .
$$

Считаем, что $N \geqslant 1$ - число шагов дискретного процесса задано.

Пусть к концу $N$-го периода требуется, чтобы на складе количество товара второго вида было не меньше заданной величины $\epsilon_{1}$ и не больше заданной величины $\epsilon_{2}$, то есть

$$
\epsilon_{1} \leqslant x_{2}(N) \leqslant \epsilon_{2} .
$$

В общем случае условие на вектор $x(N)$ может определяться спросом на производимые товары или быть планом производства.

Зададим вектор $\psi^{*}=(0 ; 1)$, тогда цель процесса управления запишется следующим образом

$$
\epsilon_{1} \leqslant\left\langle x(N), \psi^{*}\right\rangle \leqslant \epsilon_{2} .
$$

Здесь с помощью $\langle\cdot, \cdot\rangle$ обозначено скалярное произведение векторов в $\mathbb{R}^{n}$.

Окончательно задача управления запасами будет сформулирована следующим образом. Для управляемого дискретного процесса (1) определить условия на начальный запас товара $x(0)$, при которых будет выполнено (2).

3. Дискретная динамическая задача управления. Пусть задан дискретный конфликтноуправляемый процесс $[4,8,9]$

$$
x(t+1)=A(t) x(t)-f(t)+\phi,
$$

где $x \in \mathbb{R}^{n}, t=0,1, \ldots, N$.

Здесь при каждом $t=0, \ldots, N A(t)$ - матрица соответствующей размерности; $f \in F(t) \subset \mathbb{R}^{n}-$ управление, $\phi(t) \in \Phi(t) \subset \mathbb{R}^{n}$ - помеха; $F(t), \Phi(t)$ являются связными компактами.

Заданы вектор $\psi^{*}$ и числа $\alpha, \epsilon_{1}, \epsilon_{2} \in \mathbb{R}$, причем $0 \leqslant \epsilon_{1} \leqslant \epsilon_{2}$. Цель выбора управления $f$ заключается в осуществлении неравенства

$$
\epsilon_{1} \leqslant\left|\left\langle\psi^{*}, x(N)\right\rangle-\alpha\right| \leqslant \epsilon_{2} .
$$

Определим при $t=0, \ldots, N$ вектор $\psi(t)$ следующей рекуррентной формулой

$$
\psi(t)=A^{T}(t) \psi(t+1), \quad \psi(N)=\psi^{*} .
$$


Из (3) получим, что

$$
\langle\psi(t+1), x(t+1)\rangle=\langle\psi(t), x(t)\rangle-\langle\psi(t+1), f\rangle+\langle\psi(t+1), \phi\rangle .
$$

Обозначим

$$
\begin{array}{lll}
a_{-}(t)=\min _{f}\langle\psi(t+1), f\rangle, & a_{+}(t)=\max _{f}\langle\psi(t+1), f\rangle, & f \in F(t) ; \\
b_{-}(t)=\min _{f}\langle\psi(t+1), \phi\rangle, & b_{+}(t)=\max _{f}\langle\psi(t+1), \phi\rangle, & \phi \in \Phi(t) ;
\end{array}
$$

Тогда получим, что

$$
\begin{aligned}
& \langle\psi(t+1), f\rangle=\frac{a_{+}(t)+a_{-}(t)}{2}+a(t) u, \quad|u| \leqslant 1, \quad a(t)=\frac{a_{+}(t)-a_{-}(t)}{2}, \\
& \langle\psi(t+1), \phi\rangle=\frac{b_{+}(t)+b_{-}(t)}{2}+b(t) v, \quad|v| \leqslant 1, \quad b(t)=\frac{b_{+}(t)-b_{-}(t)}{2} .
\end{aligned}
$$

Определим набор чисел $\delta(t) \in \mathbb{R}, t=0, \ldots, N$

$$
\delta(t)=\delta(t+1)-\frac{a_{+}(t)+a_{-}(t)}{2}+\frac{b_{+}(t)+b_{-}(t)}{2}, \quad \delta(N)=0
$$

и перейдем к новой переменной

$$
z(t)=\langle\psi(t), x(t)\rangle-\alpha+\delta(t)
$$

Тогда управляемый процесс можно записать следующим образом

$$
z(t+1)=z(t)-a(t) u+b(t) v, \quad|u| \leqslant 1, \quad|v| \leqslant 1 .
$$

При любых числах $0 \leqslant \epsilon_{1} \leqslant \epsilon_{2}$ введем в рассмотрение кольцо

$$
S\left(\epsilon_{1} ; \epsilon_{2}\right)=\left\{z \in \mathbb{R}^{n}: \epsilon_{1} \leqslant|z| \leqslant \epsilon_{2}\right\} .
$$

Тогда цель (4) процесса управления будет

$$
z(N) \in S\left(\epsilon_{1} ; \epsilon_{2}\right) .
$$

4. Постановка задачи. Рассмотрим дискретный процесс вида

$$
z(t+1)=z(t)-a(t) u+b(t) v
$$

где $z \in \mathbb{R}^{n},|u(t)| \leqslant 1,|v(t)| \leqslant 1, a(t) \geqslant 0, b(t) \geqslant 0, t=0, \ldots, N$. Здесь $u(t) \in U(t) \subset \mathbb{R}^{n}-$ значения управления, $v(t) \in V(t) \subset \mathbb{R}^{n}$ - значения помехи. Считаем, что $N \geqslant 1$ - число шагов (длительность) процесса управления задано.

Заданы два числа $0 \leqslant \epsilon_{1} \leqslant \epsilon_{2}$. Цель управления процессом $(6)$ заключается в осуществлении включения (5) в конечный момент времени.

Управление происходит следующим образом. В начальный момент времени задано начальное состояние $z(0)$. Исходя из начального положения, происходит реализация помехи $v(0)$ из множества $V(0)$. Затем выбирается управление $u(0)$ из множества $U(0)$. Для начального условия $z(0)$ и для выбранной допустимой пары $v(0), u(0)$ по формуле $(6)$ реализуется состояние $z(1)$. Оно принимается за начальное. Далее управление строится по правилу, описанному выше.

Требуется определить множество начальных положений $z(0)$, откуда возможно осуществить (5) при любых допустимых реализациях помехи.

5. Оператор программного поглощения. Сначала введем в рассмотрение геометрическую разность двух множеств $A$ и $B$ из пространства $\mathbb{R}^{n}[3]$

$$
A \stackrel{*}{-} B=\left\{z \in \mathbb{R}^{n}: z+B \subset A\right\} .
$$

Далее обозначим

$$
S=\left\{z \in \mathbb{R}^{n}:|z| \leqslant 1\right\} .
$$

Введем оператор $T_{k}$, который каждому числу $k=0,1, \ldots, N$ и каждому множеству $Y \subset \mathbb{R}^{n}$ ставит в соответствие множество $T_{k}(Y)$, определяемое следующим образом. Точка $z \in T_{k}(Y)$ тогда 
и только тогда, когда для любого управления $v(k) \in V(k)$ существует управление $u(k) \in U(k)$ такое, что

Отсюда получим, что

$$
z(k+1)=z(k)-a(k) u(k)+b(k) v(k) \in Y .
$$

Положим, что $T_{k}(\emptyset)=\emptyset$.

$$
T_{k}(Y)=(Y+a(k) S) \stackrel{*}{-} b(k) S
$$

Обозначим

$$
\Omega_{m}=T_{m}\left(T_{m+1}\left(\ldots\left(T_{N-1}(Y)\right) \ldots\right)\right), \quad m=0,1, \ldots, N-1 .
$$

Используя введенный оператор программного поглощения, получим, что множество начальных положений, откуда первый игрок сможет осуществить включение $(5)$, запишется в виде $\Omega_{0}$.

Рассмотрим случай, когда множество $Y=S\left(\epsilon_{1} ; \epsilon_{2}\right)$. Для определения оператора программного поглощения (7) в этом случае потребуются следующие леммы [5].

Лемма 1. Для любых чисел $0 \leqslant \delta \leqslant \epsilon u \sigma \geqslant 0$ выполнено равенство

$$
S(\delta ; \epsilon)+\sigma S=S(\max (0 ; \delta-\sigma) ; \epsilon+\sigma) .
$$

Лемма 2. Для любых чисел $0 \leqslant \delta \leqslant \epsilon u \sigma \geqslant 0$ выполнено равенство

$$
S(\delta ; \epsilon) \stackrel{*}{-} \sigma S= \begin{cases}S(f(\delta ; \sigma) ; \epsilon-\sigma), & \text { npu } f(\delta ; \sigma)+\sigma \leqslant \epsilon \\ \emptyset, & \text { npu } f(\delta ; \sigma)+\sigma>\epsilon .\end{cases}
$$

где $f(0 ; \sigma)=0$ при любом $\sigma \geqslant 0 ; f(\delta ; \sigma)=\delta+\sigma$ при любых $\delta>0 u \sigma \geqslant 0$.

Найдем (7) при $0 \leqslant k \leqslant N$, когда $Y=S\left(\epsilon_{1} ; \epsilon_{2}\right)$.

Теорема 1. Для любых $0 \leqslant \epsilon_{1} \leqslant \epsilon_{2} u 0 \leqslant k \leqslant N$ верно

$$
T_{k}\left(S\left(\epsilon_{1} ; \epsilon_{2}\right)\right)= \begin{cases}S\left(h_{k}\left(\epsilon_{1}\right) ; \epsilon_{2}+a(k)-b(k)\right), & \text { при } h_{k}\left(\epsilon_{1}\right) \leqslant \epsilon_{2}+a(k)-b(k), \\ \emptyset, & \text { в противном случае. }\end{cases}
$$

где

$$
h_{k}\left(\epsilon_{1}\right)= \begin{cases}\epsilon_{1}-a(k)+b(k), & \text { при } \epsilon_{1}-a(k)>0, \\ 0, & \text { в противном случае. }\end{cases}
$$

Доказательство. Используем лемму 11, получим

$$
T_{k}\left(S\left(\epsilon_{1} ; \epsilon_{2}\right)\right)=\left(S\left(\epsilon_{1} ; \epsilon_{2}\right)+a(k) S\right) \stackrel{*}{-} b(k) S=S\left(\max \left(0 ; \epsilon_{1}-a(k)\right) ; \epsilon_{2}+a(k)\right) \stackrel{*}{-} b(k) S .
$$

Далее, применяя лемму 2 , запишем

$$
T_{k}\left(S\left(\epsilon_{1} ; \epsilon_{2}\right)\right)=\left\{\begin{array}{l}
\emptyset, \text { при } f\left(\max \left(0 ; \epsilon_{1}-a(k)\right) ; b(k)\right)>\epsilon_{2}+a(k)-b(k), \\
S\left(f\left(\max \left(0 ; \epsilon_{1}-a(k)\right) ; b(k)\right) ; \epsilon_{2}+a(k)-b(k)\right), \text { в противном случае. }
\end{array}\right.
$$

Из последнего выражения следует утверждение теоремы.

6. Нахождение множества $\Omega_{0}$. Найдем сначала множество $\Omega_{m}$.

Теорема 2. Для любого числа $0 \leqslant m \leqslant N-1$ выполнено

Здесь обозначено

$$
\Omega_{m}= \begin{cases}S\left(\gamma_{1}(m) ; \gamma_{2}(m)\right), & \text { при } \max _{m \leqslant j \leqslant N-1}\left(\gamma_{1}(j)-\gamma_{2}(j)\right)<0, \\ \emptyset, & \text { в противном случае. }\end{cases}
$$

$$
\begin{aligned}
& \gamma_{1}(m)=\left\{\begin{array}{l}
0, \text { nри } \min \left(\epsilon_{1}-a(N-1) ; \ldots ; \epsilon_{1}-\sum_{i=m}^{N-1} a(i)+\sum_{i=m+1}^{N-1} b(i)\right) \leqslant 0 \\
\epsilon_{1}-\sum_{i=m}^{N-1}(a(i)-b(i)), \text { в противном случае, }
\end{array}\right. \\
& \gamma_{2}(m)=\epsilon_{2}+\sum_{i=m}^{N-1}(a(i)-b(i)), \quad m=0, \ldots, N-1 .
\end{aligned}
$$


Доказательство. Согласно (8) множество $\Omega_{N-1}=T_{N-1}\left(S\left(\epsilon_{1} ; \epsilon_{2}\right)\right)$.

Из определения оператора $T_{k}(Y)$ и теоремы 1 получим, что

$$
\Omega_{N-1}= \begin{cases}S\left(h_{N-1}\left(\epsilon_{1}\right) ; \epsilon_{2}+a(N-1)-b(N-1)\right) & \text { при } h_{N-1}\left(\epsilon_{1}\right) \leqslant \epsilon_{2}+a(N-1)-b(N-1), \\ \emptyset & \text { в противном случае }\end{cases}
$$

где

$$
h_{N-1}\left(\epsilon_{1}\right)= \begin{cases}\epsilon_{1}-a(N-1)+b(N-1) & \text { при } \epsilon_{1}-a(N-1)>0 \\ 0 & \text { в противном случае. }\end{cases}
$$

Далее запишем $\Omega_{N-2}=T_{N-2}\left(T_{N-1}\left(S\left(\epsilon_{1} ; \epsilon_{2}\right)\right)\right)$.

Снова воспользуемся определением оператора $T_{k}(Y)$ и результатом теоремы 1.

Тогда

$$
\begin{aligned}
& \Omega_{N-2}=S\left(\max \left(0 ; h_{N-1}\left(\epsilon_{1}\right)-a(N-2)\right) ; \epsilon_{2}+a(N-1)-b(N-1)+a(N-2)\right)-b(N-2) S= \\
& =\left\{\begin{array}{c}
S\left(f\left(\max \left(0 ; h_{N-1}\left(\epsilon_{1}\right)-a(N-2)\right) ; b(N-2)\right) ;\right. \\
\left.\epsilon_{2}+a(N-1)-b(N-1)+a(N-2)-b(N-2)\right) \\
\text { при } f\left(\max \left(0 ; h_{N-1}\left(\epsilon_{1}\right)-a(N-2)\right) ; b(N-2)\right) \leqslant \\
\leqslant \epsilon_{2}+a(N-1)-b(N-1)+a(N-2)-b(N-2), \\
\text { в противном случае }
\end{array}\right. \\
& =\left\{\begin{array}{c}
S\left(\gamma_{1}(N-2) ; \gamma_{2}(N-2)\right) \text { при } \max \left(\gamma_{1}(N-1)-\gamma_{2}(N-1) ; \gamma_{1}(N-2)-\gamma_{2}(N-2)\right)<0, \\
\emptyset \text { в противном случае. }
\end{array}\right.
\end{aligned}
$$

Продолжая предыдущие рассуждения, получим утверждение теоремы.

Следовательно, для того, чтобы из начального положения $z(0)$ осуществить включение (5), должны выполняться неравенства

$$
\gamma_{1}(0) \leqslant|z(0)| \leqslant \gamma_{2}(0)
$$

при условии

где

$$
\max _{0 \leqslant j \leqslant N-1}\left(\gamma_{1}(j)-\gamma_{2}(j)\right)<0
$$

$$
\begin{aligned}
& \gamma_{1}(0)=\left\{\begin{array}{l}
0 \quad \text { при } \min \left(\epsilon_{1}-a(N-1) ; \ldots ; \epsilon_{1}-\sum_{i=0}^{N-1} a(i)+\sum_{i=1}^{N-1} b(i)\right) \leqslant 0, \\
\epsilon_{1}-\sum_{i=0}^{N-1}(a(i)-b(i)) \quad \text { в противном случае }
\end{array}\right. \\
& \gamma_{2}(0)=\epsilon_{2}+\sum_{i=0}^{N-1}(a(i)-b(i)) .
\end{aligned}
$$

\section{СПИСОК ЛИТЕРАТУРЫ}

1. Изместъев И. В., Ухоботов В. И. Об одном подходе к построению управления первого игрока в однотипной дифференциальной игре с терминальным множеством в форме кольца// Челябинск. физ.мат. ж. - 2018. - 3, № 2. - С. 144-152.

2. Никитина C. А., Скорынин А. С., Ухоботов В. И. Об одной задаче управления дискретной системой// Челябинск. физ.-мат. ж. - 2018. - 3, № 3. - С. 311-318.

3. Понтрягин Л. С. Линейные дифференциальные игры, ІІ// Докл. АН СССР. - 1967. - 175, № 4. C. $764-766$.

4. Пропой А. И. Элементы теории оптимальных дискретных процессов. - М.: Наука, 1973. 
5. Ухоботов В. И. Однотипная дифференциальная игра с терминальным множеством в форме кольца// в кн.: Некоторые задачи динамики и управления. - Челябинск: Челябинск. гос. ун-т, 2005. - С. $108-$ 123.

6. Ухоботов В. И., Изместьев И. В. Однотипная задача импульсной встречи в заданный момент времени с терминальным множеством в форме кольца// Вестн. Удмурт. ун-та. Мат. Мех. Комп. науки. - 2015. -25 , № 2. - С. 197-211.

7. Ухоботов В. И., Изместьев И. В. Синтез управлений в однотипной игровой задаче импульсной встречи в заданный момент времени с терминальным множеством в форме кольца// Вестн. Удмурт. ун-та. Мат. Мех. Комп. науки. - 2017. - 27, № 1. - С. 69-85.

8. Ухоботов В. И., Стабулит И. С. Динамическая задача управления при наличии помехи и с заданным множеством моментов коррекции// Вестн. Удмурт. ун-та. Мат. Мех. Комп. науки. -2018 . - 28, № 1 . - C. $74-81$.

9. Шориков А. Ф. Алгоритм адаптивного минимаксного управления для процесса преследованияуклонения в дискретных системах// Тр. ин-та мат. мех. УрО РАН. - 2000. - 6, № 2. - С. 515-535.

Никитина Светлана Анатольевна

Челябинский государственный университет

E-mail: nikitina@csu.ru

Ухоботов Виктор Иванович

Челябинский государственный университет

E-mail: ukh@csu.ru 